\title{
Benchmark datasets for phylogenomic pipeline validation, applications for foodborne pathogen surveillance
}

\author{
Ruth E Timme ${ }^{\text {Corresp.., }}{ }^{1}$, Hugh Rand ${ }^{1}$, Martin Shumway ${ }^{2}$, Eija K Trees ${ }^{3}$, Mustafa Simmons ${ }^{4}$, Richa Agarwala ${ }^{2}$ \\ , Steven Davis ${ }^{1}$, Glenn E Tillman ${ }^{4}$, Stephanie Defibaugh-Chavez ${ }^{5}$, Heather A Carleton ${ }^{3}$, William A Klimke ${ }^{2}$, \\ Lee S Katz ${ }^{3,6}$ \\ ${ }^{1}$ Center for Food Safety and Applied Nutrition, US Food and Drug Administration, College Park, Maryland, United States \\ 2 National Center for Biotechnology Information, National Institutes of Health, Bethesda, Maryland, United States \\ 3 Enteric Diseases Laboratory Branch, Centers for Disease Control and Prevention, Atlanta, Georgia, United States \\ 4 Food Safety and Inspection Service, US Department of Agriculture, Athens, Georgia, United States \\ 5 Food Safety and Inspection Service, US Department of Agriculture, Wahington, DC, United States \\ ${ }^{6}$ Center for Food Safety, College of Agricultural and Environmental Sciences, University of Georgia, Griffin, Georgia, United States \\ Corresponding Author: Ruth E Timme \\ Email address: ruth.timme@fda.hhs.gov
}

Background. As next generation sequence technology has advanced, there have been parallel advances in genome-scale analysis programs for determining evolutionary relationships as proxies for epidemiological relationship in public health. Most new programs skip traditional steps of ortholog determination and multi-gene alignment, instead identifying variants across a set of genomes, then summarizing results in a matrix of single-nucleotide polymorphisms or alleles for standard phylogenetic analysis. However, public health authorities need to document the performance of these methods with appropriate and comprehensive datasets so they can be validated for specific purposes, e.g., outbreak surveillance. Here we propose a set of benchmark datasets to be used for comparison and validation of phylogenomic pipelines.

Methods. We identified four well-documented foodborne pathogen events in which the epidemiology was concordant with routine phylogenomic analyses (reference-based SNP and wgMLST approaches). These are ideal benchmark datasets, as the trees, WGS data, and epidemiological data for each are all in agreement. We have placed these sequence data, sample metadata, and "known" phylogenetic trees in publicly-accessible databases and developed a standard descriptive spreadsheet format describing each dataset. To facilitate easy downloading of these benchmarks, we developed an automated script that uses the standard descriptive spreadsheet format.

Results. Our "outbreak" benchmark datasets represent the four major foodborne bacterial pathogens (Listeria monocytogenes, Salmonella enterica, Escherichia coli, and Campylobacter jejuni) and one simulated dataset where the "known tree" can be accurately called the "true tree". The downloading script and associated table files are available on GitHub: https://github.com/WGS-standards-andanalysis/datasets.

Discussion. These five benchmark datasets will help standardize comparison of current and future phylogenomic pipelines, and facilitate important cross-institutional collaborations. Our work is part of a global effort to provide collaborative infrastructure for sequence data and analytic tools - we welcome additional benchmark datasets in our recommended format, and, if relevant, we will add these on our GitHub site. Together, these datasets, dataset format, and the underlying GitHub infrastructure present a recommended path for worldwide standardization of phylogenomic pipelines. 
1 Benchmark datasets for phylogenomic pipeline validation, applications

2 for foodborne pathogen surveillance

4 Ruth E. Timme ${ }^{1}$, Hugh Rand ${ }^{1}$, Martin Shumway ${ }^{2}$, Eija K. Trees ${ }^{3}$, Mustafa Simmons ${ }^{4}$, Richa

5 Agarwala $^{2}$, Steve Davis ${ }^{1}$, Glenn Tillman ${ }^{4}$, Stephanie Defibaugh-Chávez ${ }^{5}$, Heather A. Carleton 6 , William A. Klimke ${ }^{2}$, Lee S. Katz ${ }^{3,6}$

$7 \quad{ }^{1}$ Center for Food Safety \& Applied Nutrition, U.S. Food \& Drug Administration, College Park, 8 Maryland

$9{ }^{2}$ National Center for Biotechnology Information, National Library of Medicine, National

10 Institutes of Health, Bethesda, MD, USA

$11{ }^{3}$ Enteric Diseases Laboratory Branch, Centers for Disease Control and Prevention, Atlanta,

12 Georgia

$13{ }^{4}$ U.S. Department of Agriculture, Food Safety and Inspection Service, Office of Public Health 14 Science, Athens, GA

$15{ }^{5}$ U.S. Department of Agriculture, Food Safety and Inspection Service, Office of Public Health 16 Science, Washington, D.C.

$17{ }^{6}$ Center for Food Safety, College of Agricultural and Environmental Sciences, University of

18 Georgia, Griffin, GA, USA

19 Corresponding Author: Ruth Timme ${ }^{1}$

20 Email address: ruth.timme@fda.hhs.gov 


\section{Abstract}

22 Background. As next generation sequence technology has advanced, there have been parallel

23 advances in genome-scale analysis programs for determining evolutionary relationships as

24 proxies for epidemiological relationship in public health. Most new programs skip traditional

25 steps of ortholog determination and multi-gene alignment, instead identifying variants across a

26 set of genomes, then summarizing results in a matrix of single-nucleotide polymorphisms or

27 alleles for standard phylogenetic analysis. However, public health authorities need to document

28 the performance of these methods with appropriate and comprehensive datasets so they can be

29 validated for specific purposes, e.g., outbreak surveillance. Here we propose a set of benchmark

30 datasets to be used for comparison and validation of phylogenomic pipelines.

31 Methods. We identified four well-documented foodborne pathogen events in which the 32 epidemiology was concordant with routine phylogenomic analyses (reference-based SNP and

33 wgMLST approaches). These are ideal benchmark datasets, as the trees, WGS data, and

34 epidemiological data for each are all in agreement. We have placed these sequence data, sample

35 metadata, and "known" phylogenetic trees in publicly-accessible databases and developed a

36 standard descriptive spreadsheet format describing each dataset. To facilitate easy downloading

37 of these benchmarks, we developed an automated script that uses the standard descriptive

38 spreadsheet format.

39 Results. Our "outbreak" benchmark datasets represent the four major foodborne bacterial 40 pathogens (Listeria monocytogenes, Salmonella enterica, Escherichia coli, and Campylobacter

41 jejuni) and one simulated dataset where the "known tree" can be accurately called the "true tree".

42 The downloading script and associated table files are available on GitHub:

43 https://github.com/WGS-standards-and-analysis/datasets

44 Discussion. These five benchmark datasets will help standardize comparison of current and 45 future phylogenomic pipelines, and facilitate important cross-institutional collaborations. Our

46 work is part of a global effort to provide collaborative infrastructure for sequence data and

47 analytic tools - we welcome additional benchmark datasets in our recommended format, and, if

48 relevant, we will add these on our GitHub site. Together, these datasets, dataset format, and the

49 underlying GitHub infrastructure present a recommended path for worldwide standardization of

50 phylogenomic pipelines. 


\section{Introduction}

52 Foodborne pathogen surveillance in the United States is currently undergoing an important

53 paradigm shift: pulsed-field gel electrophoresis (PFGE) (Swaminathan et al., 2001) is being

54 replaced by the much higher resolution whole genome sequencing (WGS) technology. The data

55 are also more accessible as the raw genome data are now being made public immediately after

56 collection. These advances began with an initial pilot project to build a public genomic reference

57 database, "GenomeTrakr" (Allard et al., 2016) for pathogens from the food supply and has

58 matured through a second pilot project to collect WGS data and share it publicly in real time for

59 every Listeria monocytogenes isolate appearing in the US food supply (both clinical and

60 food/environmental isolates) (Jackson et al., 2016). The Real-Time Listeria Project was initiated

61 by PulseNet, the national subtyping network for foodborne disease surveillance, and is

62 coordinated by the Centers for Disease Control and Prevention (CDC), the Food and Drug

63 Administration (FDA), the National Center for Biotechnology Information (NCBI), and the Food

64 Safety and Inspection Service (FSIS) of the United States Department of Agriculture. The success

65 of the project confirmed that a national laboratory surveillance program using WGS is possible

66 and highly efficient. Now, genome data are collected in real-time for five major bacterial

67 foodborne pathogens (Salmonella enterica, Listeria monocytogenes, Escherichia coli, Vibrio

68 parahaemolyticus and Campylobacter spp.); WGS data are being deposited in either the

69 Sequence Read Archive (SRA) or GenBank, and are being clustered into phylogenetic trees using

70 SNP analysis; results are publicly available at NCBI's pathogen detection web site (). The list of

71 pathogens under active genomic surveillance is growing. As of August $16^{\text {th }}, 2017$, over 150

72 thousand genomes have been sequenced and contributed towards this public pathogen

73 surveillance effort.

74 The collaboration among the FDA, NCBI, FSIS, and CDC has been formalized as the Genomics 75 and Food Safety group (Gen-FS) 
$76(\mathrm{CDC}, 2015)$

77 . One of the first directives for Gen-FS is ensuring consistency across the different tools for 78 phylogenomic analysis used by group participants. The best way to accomplish this is to have 79 standard benchmark datasets, which enable researchers to assess the consistency of results across 80 different tools and between version updates of any single tool. Each agency has been using 81 compatible bioinformatics workflows for their WGS analysis. PulseNet-participating laboratories

82 use whole genome multilocus sequence typing (wgMLST) with core-genome multilocus

83 sequence typing (cgMLST) at its core (Moura et al., 2016). NCBI uses the Pathogen Detection

84 Pipeline (Available at https://www.ncbi.nlm.nih.gov/pathogens). At the FDA, the Center for Food

85 Safety and Applied Nutrition (CFSAN) uses SNP-Pipeline (Davis et al., 2015). The CDC uses

86 Lyve-SET (Katz et al., 2017). These methods have been designed to match the specific needs of

87 the different agencies performing bacterial foodborne pathogen surveillance. For example,

88 PulseNet surveillance identifies clusters of closely related clinical isolates from cases of

89 foodborne disease that may be followed up in outbreak investigations by all three agencies. After

90 the WGS and epidemiological evidence are considered the FDA and FSIS conduct further

91 investigations and take appropriate regulatory actions. Other phylogenomic analysis packages

92 could also benefit from standardized benchmark datasets, e.g., NASP, Harvest, kSNPv3,

93 REALPHY and SNVPhyl (Gardner \& Hall, 2013; Treangen et al., 2014; Bertels et al., 2014; Sahl

94 et al., 2016; Petkau et al., 2017). Consistent validation of the many available analysis packages is

95 essential if we are to use genomic data for regulatory action.

96 Many pathogen outbreak datasets with raw reads have been made public, for example, genomes

97 from several North American Listeria monocytogenes events (Chen et al., 2016; 2017b,a) a

98 Yersinia pestis outbreak from North America (Sahl et al., 2016), a Clostridioides difficile

99 outbreak dataset from the UK (Treangen et al., 2014), a Clostridioides difficile outbreak in the 
100 UK (Eyre et al., 2013), the S. enterica subsp. enterica serovar Bareilly (S. enterica ser Bareilly)

1012012 outbreak in the US (Hoffmann et al., 2015), and an S. enterica subsp. enterica serovar

102 Enteritidis outbreak in the UK (Quick et al., 2015). Additionally, many datasets have been

103 published during the course of peer review for this paper, making it difficult to keep track of all

104 datasets. However, they are not in a standardized format, making them difficult to acquire or use

105 in automated analyses. As of November 2016, no bacterial outbreak datasets have been

106 specifically published for use as benchmark datasets. Here we present a set of outbreak

107 benchmark datasets for use in comparison and validation of phylogenomic pipelines.

108 Materials \& Methods

109 We present one empirical dataset for each of four major foodborne bacterial pathogens $(L$.

110 monocytogenes, $S$. enterica ser. Bareilly, E. coli, and C. jejuni) and one simulated dataset

111 generated from the S. enterica ser. Bareilly tree using the pipeline TreeToReads (McTavish et al.,

112 2017), for which both the true tree and SNP positions are known. In addition, we propose a

113 standard spreadsheet format for describing these and future benchmark datasets. That format can

114 be readily applied to any other bacterial organism and supports automated data analyses. Finally,

115 we present Gen-FS Gopher, a script for easily downloading these benchmark datasets. All of

116 these materials are freely available for download at the GitHub site:

117 URL: https://github.com/WGS-standards-and-analysis/datasets

118 Each of the four empirical datasets is either representative of a food recall event in which food

119 was determined to be contaminated with a specific bacterial pathogen, or of an outbreak in which

120 at least three people were infected with the same pathogen. In all four datasets, the results of the

121 epidemiological investigation and the phylogenomic analyses are in concordance. In other words,

122 all isolates implicated in a given event share a common ancestor, or cluster together, in the

123 phylogeny. Although it might be tempting to place these four datasets in the context of a

124 transmission network, it is not the appropriate usage. A phylogeny (with clinical and

125 environmental isolates at the tips and inferred ancestors at internal nodes) is more appropriate due

126 to the nature of foodborne outbreaks: point sources that usually originate from food vehicles,

127 whereas a transmission network more appropriately models person-to-person transmission

128 events. Although our particular four datasets are not intended for transmission network analysis,

129 this does not prevent any future datasets with this intended usage. On the contrary, we have

130 included a field "intendedUse" which addresses this issue and helps future-proof the proposed 
131 dataset format (Table 1). All isolates listed in these benchmark datasets were sequenced at our

132 federal or state-partner facilities, using either an Illumina MiSeq (San Diego, CA) or a Pacific

133 Biosciences (PacBio) instrument (Menlo Park, CA).

134 The simulated dataset was created using the TreeToReads v 0.0.5 (McTavish et al., 2017), which

135 takes as input a tree file (true phylogeny), an anchor genome, and a set of user-defined parameter

136 values. We used the $S$. enterica ser. Bareilly tree as our "true" phylogeny and the closed reference

137 genome (CFSAN000189, GenBank: GCA_000439415.1) as our anchor. The parameter values

138 were set as follows: number_of_variable_sites = 150, base_genome_name =

139 CFSAN000189, rate_matrix $=0.38,3.83,0.51,0.01,4.45,1$, freq_matrix

$140=0.19,0.30,0.29,0.22$, coverage $=40$, mutation_clustering $=$ ON,

141 percent_clustered $=0.25$, exponential_mean $=125$, read_length $=250$,

142 fragment_size $=500$, stdev_frag_size $=120$. The output is a pair of raw MiSeq

143 fastq files for each tip (simulated isolate) in the input tree and a VCF file of known SNP

144 locations.

145 Maximum likelihood phylogenies included for each dataset were inferred by first gathering SNPs

146 from SNP Pipeline (Davis et al., 2015) and then using Garli version 2.01 (Zwickl, 2006) for

147 phylogenetic reconstruction on each resulting SNP matrix.

\section{Results/Discussion}

149 The L. monocytogenes dataset (Supplemental Table S1) comprises genomes spanning the genetic

150 diversity of the 2014 stone fruit recall (Jackson et al., 2016; Chen et al., 2016). In this event, a

151 company voluntarily recalled certain lots of stone fruits (peaches and the like) based on the

152 company's internal tests, which were positive for the presence of L. monocytogenes. This dataset

153 describes a polyclonal phylogeny having three major subclades, two of which include clinical

154 cases. The genome for one isolate was closed, yielding a complete reference genome. This

155 dataset also includes three outgroups that were not associated with the outbreak.

156 The C. jejuni dataset (Supplemental Table S2) represents a 2008 outbreak in Pennsylvania

157 associated with raw milk (Marler, 2008). This dataset reflects a clonal outbreak lineage with

158 several outgroups not related to the outbreak strain. 
159 The E. coli dataset (Supplemental Table S3) is from a 2014 outbreak in which raw clover sprouts

160 were identified as the transmission vehicle (CDC, 2014). Nineteen clinical cases had the same

161 clone of Shiga-toxin-producing E. coli O121. The genome for one isolate that was

162 epidemiologically unrelated to the outbreak but phylogenetically related was closed, yielding a

163 complete reference genome. Only three of the available 19 clinical isolates were included in this

164 dataset; these isolates were so highly clonal that adding more genomes from the outbreak would

165 not provide additional insights. This dataset also includes seven closely related outgroup isolates

166 that were not part of the outbreak.

167 A S. enterica ser. Bareilly dataset (Supplemental Table S4) was derived from a 2012 outbreak in 168 mid-Atlantic US states associated with spicy tuna sushi rolls (CDC, 2012). Both epidemiological 169 data and WGS data indicate that patients in the United States became infected with S. enterica

170 ser. Bareilly by consuming tuna scrape that had been imported for making spicy tuna sushi from a 171 fishery in India (Hoffmann et al., 2015). This benchmark dataset includes 18 clonal outbreak 172 taxa, comprising both clinical and food isolates. Five outgroups are also included in this dataset, 173 one of which was closed and serves as the reference genome.

174 The simulated dataset (Supplemental Table S5) was generated from the empirical Salmonella 175 phylogeny described above. This dataset is useful for validating the number and location of SNPs 176 identified from a given bioinformatics pipeline and can help measure exactly how close an 177 inferred phylogeny is to the true phylogeny since the "true" phylogeny is known in this case.

178 This dataset comprises 18 simulated outbreak isolates and five outgroups, mirroring the empirical 179 tree.

\section{The dataset format}

181 Tables 1 and 2 list the standardized descriptions used in each dataset, beginning with the required $182 \mathrm{key} / \mathrm{value}$ pairs, followed by the available field names. Table 3 illustrates the use of this 183 standardized reporting structure: columns in this format provide accession numbers for the 184 sequence and phylogenetic tree data. Columns also contain epidemiological data characterizing 185 the isolate as inside or outside of that specific outbreak. These data are housed at NCBI, a partner 186 of the International Nucleotide Sequence Database Collaboration (INSDC) (Karsch-Mizrachi et 187 al., 2012), and at OpenTree (Hinchliff et al., 2015). The tree topologies provided for each dataset 188 (Figure 1) were robust to different phylogenomic pipelines, such Lyve-Set (another SNP-based 
189 pipeline) (Katz et al., 2017) and wgMLST (allele-based pipeline) (Moura et al., 2016). To the

190 best of our knowledge, the tree accompanying each dataset closely represents the true phylogeny,

191 given the current taxon sampling and accepted epidemiology. For each benchmark dataset we

192 include the following data:

193 1. NCBI Sequence Read Archive (SRA) accessions for each isolate.

194 2. NCBI BioSample accession for each isolate.

195 3. A link to a maximum likelihood phylogenetic tree stored at the OpenTreeOfLife

196 4. NCBI assembly accessions for annotated draft and complete assemblies (where available).

197 Information is provided about which assembly is appropriate for use as a reference.

198 The benchmark table format is a spreadsheet divided into two sections: a header and the body.

199 The header contains generalized information of the dataset in a key/value format where column A

200 is the key and the value is in column B. The available keys with example values are given in

201 Table 1. Any property in the header applies to all genomes; for example, all isolates described in

202 the spreadsheet should be of the same organism as listed in the header. The body of the dataset

203 provides information for each taxon, or tip in the tree. Accessions, strain IDs, key to isolates in

204 clonal event, and sha256sums are included here (Table 2). An example is given in Table 3.

205 To ensure that every dataset is easily and reliably downloadable for anyone to use, we have

206 created a script called Gen-FS Gopher (GG) that automates the download process. GG

207 downloads the assemblies, raw reads, and tree(s) listed in a given dataset spreadsheet.

208 Additionally, GG uses the sha256sum program to verify each download. Because some files

209 depend on others (e.g., downloading the reverse read depends on the forward read; the

210 sha256sha256 checksums depend on all reads being downloaded), GG creates a Makefile, which

211 is then executed. That Makefile creates a dependency tree such that all files will be downloaded

212 in the order they are needed. Each of our five benchmark datasets, described in Table 4, can be

213 downloaded using this GG script.

\section{Conclusion}

215 The analysis and interpretation of datasets at the genomic scale is challenging due to the volume

216 of data as well as the complexity and number of software programs often involved in the process. 
217 To have confidence in such analyses, it is important to be able to verify the performance of

218 methods against datasets where the answers are already known. Ideally, such datasets provide a

219 basis for not just testing methods, but also helping to provide a basis for ensuring the

220 reproducibility of new methods and establishing comparability between bioinformatics pipelines.

221 Having an established table format and tools to ensure easy and accurate downloads of

222 benchmark datasets will help codify how data can be shared and evaluated. Here we have

223 described five such datasets relevant for bacterial foodborne investigations based on WGS data.

224 We have also established a standard file format suitable for these and future benchmark datasets,

225 along with a script that is able to read and properly download them. It is to be emphasized that

226 these benchmark datasets are useful for comparisons of phylogenomic pipelines and do not

227 replace a more extensive validation of new pipelines. Such a new pipeline must be validated for

228 typability, reproducibility, repeatability, discriminatory power, and epidemiological concordance

229 using extensive isolate collections that are representative for the correct epidemiological context

230 (van Belkum et al., 2007).

231 The Gen-FS Gopher script along with five new benchmark datasets encourages reproducibility in

232 the rapidly growing field of phylogenomics for pathogen surveillance. Currently, when new

233 datasets are published the accessions to each data piece are embedded in a table within the body

234 of the manuscript. Extracting these accessions from a PDF file can be arduous for large datasets.

235 Without the GG script one would have to write their own program for downloading data from

236 multiple databases (BioSample, SRA, GenBank, Assembly database at NCBI, and

237 OpenTreeOfLife) or manually browse each database using cut/paste operations for each

238 accession, downloading one by one. Using either route, the end result is often a directory of

239 unorganized files and inconsistent file names, requiring tedious hand manipulation to get the

240 correct file names and structure set up for local analysis. Because any given table of data is not in

241 a standardized format, this process becomes a one-off, and the process has to be onerously

242 reinvented for each table. Each step of this manual process increases the risk for error and

243 degrades reproducibility. Our datasets and download script democratize this process: a single

244 command can be cut/pasted into a unix/linux terminal, resulting in the automated download of the

245 entire dataset (tree, raw fastq files, and assembly files) organized correctly for downstream

246 analysis. 
247 Further experimental validation of these and future empirical datasets will strengthen this

248 resource. We will continue to work on these datasets using Sanger-sequence validation and will

249 encourage future submitters to validate their datasets, too. Additionally, we encourage future

250 submitters to make their entire datasets available through INSDC and OpenTree in our

251 recommended format. The participants in Gen-FS are also starting a collaboration with the

252 Global Microbial Identifier Program (“Global Microbial Identifier," 2011) that goes beyond the

253 annual GMI Proficiency Test. Researchers from around the world will be encouraged to

254 contribute validated empirical and simulated datasets, providing a more diverse set of benchmark

255 datasets. To aid in quality assurance, we suggest a minimum of 20x coverage for each genome in

256 a dataset. Submissions following our described spreadsheet format will ensure compatibility with

257 our download script, and should include isolates with as much BioSample metadata as possible

258 including values such as the outbreak code and isolate source (e.g., clinical or

259 food/environmental). Our work will allow other researchers to contribute benchmark datasets for

260 testing and comparing bioinformatics pipelines, which will contribute to more robust and reliable

261 analyses of genomic diversity. The GitHub page for that effort can be accessed here:

262 https://github.com/globalmicrobialidentifier-WG3/datasets.

263 Acknowledgements

264 We would like to thank Chris Tillman at CFSAN and Cheryl Tarr at CDC for sequencing work on 265 L. monocytogenes. We would also like to thank Collette Fitzgerald, Vikrant Dutta, Janet

266 Pruckler, and Grant Williams from CDC in helping identify and sequence the isolates from a well 267 understood Campylobacter outbreak. Additionally, Andre Weltman and Lisa Dettinger from the

268 Pennsylvania Department of Health gave vital information pertaining to the Campylobacter

269 outbreak. We would like to acknowledge Philip Bronstein at FSIS-USDA for his efforts. Lastly, 270 we would like to acknowledge Lili Fox Vélez from FDA for scientific writing support. 
271

272

273

274

275

276

277

278

279

280

281

282

283

284

285

286

287

288

289

290

291

292

293

294

295

296

297

298

299

300

301

302

303

304

305

306

307

308

309

310

311

312

313

314

315

316

\section{References}

Allard MW, Strain E, Melka D, Bunning K, Musser SM, Brown EW, Timme R 2016. The Practical value of Food Pathogen Traceability through Building a Whole-Genome

Sequencing Network and database. Journal of Clinical Microbiology:JCM.00081-16. DOI: 10.1128/JCM.00081-16.

Bertels F, Silander OK, Pachkov M, Rainey PB, van Nimwegen E 2014. Automated Reconstruction of Whole-Genome Phylogenies from Short-Sequence Reads. Molecular biology and evolution 31:1077-1088. DOI: 10.1093/molbev/msu088.

CDC 2012.Multistate Outbreak of Salmonella Bareilly and Salmonella Nchanga Infections Associated with a Raw Scraped Ground Tuna Product (Final Update). Available at https://www.cdc.gov/salmonella/bareilly-04-12/ (accessed December 1, 2016).

CDC 2014.Multistate Outbreak of Shiga toxin-producing Escherichia coli O121 Infections Linked to Raw Clover Sprouts (Final Update). Available at https://www.cdc.gov/ecoli/2014/o121-05-14/index.html (accessed December 1, 2016).

CDC 2015. Annual Report to the Secretary, Department of Health and Human Services. Center for Disease Control and Prevention.

Chen Y, Burall LS, Luo Y, Timme R, Melka D, Muruvanda T, Payne J, Wang C, Kastanis G, Maounounen-Laasri A, De Jesus AJ, Curry PE, Stones R, KAluoch O, Liu E, Salter M, Hammack TS, Evans PS, Parish M, Allard MW, Datta A, Strain EA, Brown EW 2016. Listeria monocytogenes in Stone Fruits Linked to a Multistate Outbreak: Enumeration of Cells and Whole-Genome Sequencing. Applied and Environmental Microbiology 82:70307040. DOI: 10.1128/AEM.01486-16.

Chen Y, Luo Y, Carleton H, Timme R, Melka D, Muruvanda T, Wang C, Kastanis G, Katz LS, Turner L, Fritzinger A, Moore T, Stones R, Blankenship J, Salter M, Parish M, Hammack TS, Evans PS, Tarr CL, Allard MW, Strain EA, Brown EW 2017a. Whole genome and core genome multilocus sequence typing and single nucleotide polymorphism analyses of Listeria monocytogenes associated with an outbreak linked to cheese, United States, 2013. Applied and Environmental Microbiology:AEM.00633-17. DOI: 10.1128/AEM.00633-17.

Chen Y, Luo Y, Curry P, Timme R, Melka D, Doyle M, Parish M, Hammack TS, Allard MW, Brown EW, Strain EA 2017b. Assessing the genome level diversity of Listeria monocytogenes from contaminated ice cream and environmental samples linked to a listeriosis outbreak in the United States. PloS one 12:e171389. DOI: 10.1371/journal.pone.0171389.

Davis S, Pettengill JB, Luo Y, Payne J, Shpuntoff Al, Rand H, Strain E 2015. CFSAN SNP Pipeline: an automated method for constructing SNP matrices from next-generation sequence data. PeerJ Computer Science 1:e20. DOI: 10.7717/peerj-cs.20.

Eyre DW, Cule ML, Wilson DJ, Griffiths D, Vaughan A, O'Connor L, Ip CLC, Golubchik T, Batty EM, Finney JM, Wyllie DH, Didelot X, Piazza P, Bowden R, Dingle KE, Harding RM, Crook DW, Wilcox MH, Peto TEA, Walker AS 2013. Diverse sources of C. difficile infection identified on whole-genome sequencing. The New England journal of medicine 369:11951205. DOI: 10.1056/NEJMoa1216064.

Gardner SN, Hall BG 2013. When Whole-Genome Alignments Just Won't Work: kSNP v2 Software for Alignment-Free SNP Discovery and Phylogenetics of Hundreds of Microbial Genomes. PloS one 8:e81760. DOI: 10.1371/journal.pone.0081760.

Hinchliff CE, Smith SA, Allman JF, Burleigh JG, Chaudhary R, Coghill LM, Crandall KA, Deng J, Drew BT, Gazis R, Gude K, Hibbett DS, Katz LA, Laughinghouse HD, McTavish EJ, 
317

318

319

320

321

322

323

324

325

326

327

328

329

330

331

332

333

334

335

336

337

338

339

340

341

342

343

344

345

346

347

348

349

350

351

352

353

354

355

356

357

358

359

360

361

362

363
Midford PE, Owen CL, Ree RH, Rees JA, Soltis DE, Williams T, Cranston KA 2015. Synthesis of phylogeny and taxonomy into a comprehensive tree of life. Proceedings of the National Academy of Sciences of the United States of America 112:12764-12769. DOI: 10.1073/pnas.1423041112.

Hoffmann M, Luo Y, Monday SR, Gonzales-Escalona N, Ottesen AR, Muruvanda T, Wang C, Kastanis G, Keys C, Janies D, Senturk IF, Catalyurek UV, Wang H, Hammack TS, Wolfgang WJ, Schoonmaker-Bopp D, Chu A, Myers R, Haendiges J, Evans PS, Meng J, Strain EA, Allard MW, Brown EW 2015. Tracing Origins of the Salmonella Bareilly strain causing a Foodborne Outbreak in the United States. The Journal of infectious diseases. DOI: 10.1093/infdis/jiv297.

Jackson BR, Tarr C, Strain E, Jackson KA, Conrad A, Carleton H, Katz LS, Stroika S, Gould LH, Mody RK, Silk BJ, Beal J, Chen Y, Timme R, Doyle M, Fields A, Wise M, Tillman G, Defibaugh-Chavez S, Kucerova Z, Sabol A, Roache K, Trees E, Simmons M, Wasilenko J, Kubota K, Pouseele H, Klimke W, Besser J, Brown E, Allard M, Gerner-Smidt P 2016. Implementation of Nationwide Real-time Whole-genome Sequencing to Enhance Listeriosis Outbreak Detection and Investigation. Clinical infectious diseases : an official publication of the Infectious Diseases Society of America 63:380-386. DOI: 10.1093/cid/ciw242.

Karsch-Mizrachi I, Nakamura Y, Cochrane G, International Nucleotide Sequence Database Collaboration 2012. The International Nucleotide Sequence Database Collaboration. Nucleic acids research 40:D33-7. DOI: 10.1093/nar/gkr1006.

Katz LS, Griswold T, Williams-Newkirk AJ, Wagner D, Petkau A, Sieffert C, Van Domselaar G, Deng X, Carleton HA 2017. A Comparative Analysis of the Lyve-SET Phylogenomics Pipeline for Genomic Epidemiology of Foodborne Pathogens. Frontiers in microbiology 8:455. DOI: 10.3389/fmicb.2017.00375.

Marler C 2008. Hendricks' Farm and Dairy Raw Milk.

McTavish EJ, Pettengill J, Davis S, Rand H, Strain E, Allard M, Timme RE 2017. TreeToReads a pipeline for simulating raw reads from phylogenies. BMC bioinformatics 18:178. DOI: 10.1186/s12859-017-1592-1.

Moura A, Criscuolo A, Pouseele H, Maury MM, Leclercq A, Tarr C, Björkman JT, Dallman T, Reimer A, Enouf V, Larsonneur E, Carleton H, Bracq-Dieye H, Katz LS, Jones L, Touchon M, Tourdjman M, Walker M, Stroika S, Cantinelli T, Chenal-Francisque V, Kucerova Z, Rocha, Eduardo P. C., Nadon C, Grant K, Nielsen EM, Pot B, Gerner-Smidt P, Lecuit M, Brisse S 2016. Whole genome-based population biology and epidemiological surveillance of Listeria monocytogenes. Nature microbiology 2:16185. DOI: 10.1038/nmicrobiol.2016.185.

Petkau A, Mabon P, Sieffert C, Knox NC, Cabral J, Iskander M, Iskander M, Weedmark K, Zaheer R, Katz LS, Nadon C, Reimer A, Taboada E, Beiko RG, Hsiao W, Brinkman F, Graham M, Van Domselaar G 2017. SNVPhyl: a single nucleotide variant phylogenomics pipeline for microbial genomic epidemiology. Microbial Genomics 3. DOI: 10.1099/mgen.0.000116.

Quick J, Ashton P, Calus S, Chatt C, Gossain S, hawker J, Nair GB, Neal K, Nye K, Peters T, De Pinna E, Robinson KS, Struthers K, Webber M, Catto A, Dallman T, Hawkey PM, Loman NJ 2015. Rapid draft sequencing and real-time nanopore sequencing in a hospital outbreak of Salmonella. Genome biology 16.

Sahl JW, Lemmer D, Travis J, Schupp JM, Gillece JD, Aziz M, Driebe EM, Drees KP, Hicks ND, Williamson CHD, Hepp CM, Smith DE, Roe C, Engelthaler DM, Wagner DM, Keim P 2016. NASP: an accurate, rapid method for the identification of SNPs in WGS datasets that supports flexible input and output formats. Microbial Genomics 2:e000074. DOI: 
364

365

366

367

368

369

370

371

372

373

374

375

376

377

378

379

380

381

382

383

384

385

386

387

388

389

390

391

392

393

394

395

396

397

10.1099/mgen.0.000074.

Swaminathan B, Barrett TJ, Hunter SB, Tauxe RV, CDC PulseNet Task Force 2001. PulseNet: the molecular subtyping network for foodborne bacterial disease surveillance, United States. Emerging Infectious Diseases 7:382-389. DOI: 10.3201/eid0703.010303.

Treangen TJ, Ondov BD, Koren S, Phillippy AM 2014. The Harvest suite for rapid core-genome alignment and visualization of thousands of intraspecific microbial genomes. Genome biology 15:524. DOI: 10.1186/PREACCEPT-2573980311437212.

van Belkum A, Tassios PT, Dijkshoorn L, Haeggman S, Cookson B, Fry NK, Fussing V, Green J, Feil E, Gerner-Smidt P, Brisse S, Struelens M, European Society of Clinical Microbiology and Infectious Diseases (ESCMID) Study Group on Epidemiological Markers (ESGEM) 2007. Guidelines for the validation and application of typing methods for use in bacterial epidemiology. Clinical Microbiology and Infection 13 Suppl 3:1-46. DOI: 10.1111/j.14690691.2007.01786.x.

Zwickl D 2006. Genetic algorithm approaches for the phylogenetic analysis of large biological sequence datasets under the maximum likelihood criterion. The University of Texas at Austin.

Global Microbial Identifier 2011. Global Microbial Identifier.

Pathogen Detection Homepage. Available at https://www.ncbi.nlm.nih.gov/pathogens/ (accessed August 16, 2017).

Tables

Table 1. Metadata table header. Available key/value pairs that describe the entire dataset.

Organism and source are required but other key/value pairs are optional.

Table 2. Metadata table body. Fields included in the body of the metadata table that describe the individual sequences included in the dataset. The required fields are biosample_acc, strain, and sra_acc. Any optional field can be blank or contain a dash (-) if no value is given. Field names are case insensitive.

Table 3. Example dataset. This as an example metadata table for a hypothetical single-isolate dataset, combining the header and body from Tables 1 and 2 .

Table 4. Benchmark datasets. The key features of the four empirical and one simulated dataset are summarized in this table.

\section{Figures}

Figure 1. The "true" phylogeny included for each dataset. The outbreak or event-related taxa are colored red. a) Listeria monocytogenes, b) Escherichia coli, c) Salmonella enterica, d) Campylobacter jejuni, e) simulated dataset. 
398 Supplemental Tables

399 Supplemental Table S1

400 Supplemental Table S2

401 Supplemental Table S3

402 Supplemental Table S4

403 Supplemental Table S5 


\section{Table $\mathbf{1}$ (on next page)}

Metadata table header

Available key/value pairs that describe the entire dataset. Organism and source are required but other key/value pairs are optional. 
Table 1. Available key/value pairs in the head of a dataset. Organism and source are required but other key/value pairs are optional.

\begin{tabular}{|c|c|c|}
\hline Key & Description & Example value(s) \\
\hline Organism & The genus, species, or other taxonomic description & Listeria monocytogenes \\
\hline Outbreak & $\begin{array}{l}\text { Usually the PulseNet outbreak code, but any other } \\
\text { descriptive word with no spaces }\end{array}$ & 1408MLGX6-3WGS \\
\hline PMID & The Pubmed identifier of a related publication & 25789745 \\
\hline Tree & The URL to a newick-formatted tree & http://api.opentreeoflife.org/v2/study/ot_301/tree/tree2.tre \\
\hline Source & A person who can be contacted about this dataset & Cheryl Tarr \\
\hline DataType & Either empirical or simulated & Empirical \\
\hline IntendedUse & $\begin{array}{l}\text { Why this dataset might be useful for someone in } \\
\text { bioinformatics testing }\end{array}$ & Epidemiologically and laboratory confirmed outbreak with outgroups \\
\hline
\end{tabular}

4 


\section{Table 2 (on next page)}

Metadata table body

Fields included in the body of the metadata table that describe the individual sequences included in the dataset. The required fields are biosample_acc, strain, and sra_acc. Any optional field can be blank or contain a dash (-) if no value is given. Field names are case insensitive. 
Table 2. Available field names for the body of a dataset. The required fields are biosample_acc, strain, and sra_acc. Any optional field can be blank or contain a dash (-) if no value is given. Field names are case insensitive.

\begin{tabular}{|c|c|c|c|}
\hline Field & Description & required & Example value(s) \\
\hline biosample_acc & $\begin{array}{l}\text { The identifier found in the NCBI BioSample database. } \\
\text { This usually starts with SAMN or SAME. }\end{array}$ & Yes & SAMN01939119 \\
\hline Strain & The name of the isolate & Yes & CFSAN002349 \\
\hline genBankAssembly & The GenBank assembly identifier & No & GCA_001257675.1 \\
\hline SRArun_acc & The Sequence Read Archive identifier & Yes & SRR1206159 \\
\hline outbreak & $\begin{array}{l}\text { If the isolate is associated with the outbreak or } \\
\text { recall, list the PulseNet outbreak code, or other } \\
\text { event identifier here. }\end{array}$ & No & $\begin{array}{l}\text { 1408MLGX6-3WGS } \\
\text { outgroup }\end{array}$ \\
\hline datasetname & To which dataset this isolate belongs & Yes & 1408MLGX6-3WGS \\
\hline suggestedReference & $\begin{array}{l}\text { For reference-based pipelines, a dataset can suggest } \\
\text { which reference assembly to use }\end{array}$ & Yes & $\begin{array}{l}\text { TRUE } \\
\text { FALSE }\end{array}$ \\
\hline sha256sumAssembly & $\begin{array}{l}\text { The sha } 256 \text { checksum of the genome assembly. This } \\
\text { will help assure that the download is successful. }\end{array}$ & Yes & $\begin{array}{l}\text { 9b926bc0adbea331a0a71f7bf18f6c7a62ebde7d } \\
\text { d7a52fabe602ad8b00722c56 }\end{array}$ \\
\hline sha256sumRead1 & The sha256 checksum of the forward read & Yes & $\begin{array}{l}\text { c43c41991ad8ed40ffcebbde36dc9011f471dea6 } \\
\text { 43fc8f715621a2e336095bf5 }\end{array}$ \\
\hline sha256sumRead2 & The sha256 checksum of the reverse read & Yes & $\begin{array}{l}\text { 4d12ed7e34b2456b8444dd71287cbb83b9c45bd } \\
\text { 18dc23627af0fbb6014ac0fca }\end{array}$ \\
\hline
\end{tabular}

4 


\section{Table 3(on next page)}

\section{Example dataset}

This is an example metadata table for a hypothetical single-isolate dataset, combining the header and body from Tables 1 and 2 . 
1 Table 3. Example dataset. This dataset compiles information from Table 1 and Table 2 and serves as an example for a hypothetical 2 single-isolate dataset.

\begin{tabular}{|c|c|c|c|c|c|c|c|c|c|}
\hline Organism & \multicolumn{9}{|c|}{ Listeria monocytogenes } \\
\hline Outbreak & \multicolumn{9}{|c|}{ 1408MLGX6-3WGS } \\
\hline PMID & \multicolumn{9}{|l|}{25789745} \\
\hline Tree & \multicolumn{9}{|c|}{ http://api.opentreeoflife.org/v2/study/ot_301/tree/tree2.tre } \\
\hline Source & \multicolumn{9}{|c|}{ Cheryl Tarr } \\
\hline DataType & \multicolumn{9}{|l|}{ Empirical } \\
\hline IntendedUse & \multicolumn{9}{|c|}{ Epi-validated outbreak } \\
\hline biosample_acc & Strain & genBankAssembly & SRArun_acc & outbreak & datasetname & $\begin{array}{l}\text { suggested } \\
\text { Reference }\end{array}$ & $\begin{array}{l}\text { sha256sum } \\
\text { Assembly }\end{array}$ & $\begin{array}{l}\text { sha256sum } \\
\text { Read1 }\end{array}$ & $\begin{array}{l}\text { sha256sum } \\
\text { Read2 }\end{array}$ \\
\hline SAMN01939119 & CFSAN002349 & GCA_001257675.1 & SRR1206159 & $\begin{array}{l}\text { 1408MLGX6- } \\
\text { 3WGS }\end{array}$ & $\begin{array}{l}\text { 1408MLGX6- } \\
\text { 3WGS }\end{array}$ & TRUE & $\begin{array}{l}9 b 926 b c 0 a \\
\text { dbea331a0 } \\
\text { a71f7bf18f } \\
6 \mathrm{c} 7 a 62 e b d \\
\text { e7dd7a52f } \\
\text { abe602ad8 } \\
\text { b00722c56 }\end{array}$ & $\begin{array}{l}\text { c43c41991 } \\
\text { ad8ed40ffc } \\
\text { ebbde36dc } \\
9011 \mathrm{f} 471 \mathrm{~d} \\
\text { ea643fc8f7 } \\
15621 \mathrm{a} 2 \mathrm{e} 3 \\
36095 \mathrm{bf5}\end{array}$ & $\begin{array}{l}\text { 4d12ed7e3 } \\
4 b 2456 b 84 \\
44 d d 71287 c \\
\text { bb83b9c45b } \\
\text { d18dc23627 } \\
\text { afOfbb6014 } \\
\text { ac0fca }\end{array}$ \\
\hline
\end{tabular}




\section{Table 4 (on next page)}

Benchmark datasets

The key features of the four empirical and one simulated dataset are summarized in this table. 
Table 4. Key dataset characteristics. The key features of each dataset are given in this table.

\begin{tabular}{|c|c|c|c|c|c|c|}
\hline Dataset & Organism & $\begin{array}{l}\text { Number of } \\
\text { Isolates a }\end{array}$ & $\begin{array}{l}\text { Epidemiologically } \\
\text { linked Isolates }{ }^{\text {b }}\end{array}$ & $\begin{array}{l}\text { reference } \\
\text { genome }^{c}\end{array}$ & $\begin{array}{l}\text { Type of } \\
\text { dataset }\end{array}$ & Reference/Comment \\
\hline $\begin{array}{l}\text { Stone Fruit Food } \\
\text { recall }\end{array}$ & L. monocytogenes & 31 & 28 & CFSAN023463 & Empirical & PMID: 27694232 \\
\hline $\begin{array}{l}\text { Spicy Tuna } \\
\text { outbreak }\end{array}$ & S. enterica & 23 & 18 & CFSAN000189 & Empirical & PMID: 25995194 \\
\hline $\begin{array}{l}\text { Raw Milk } \\
\text { Outbreak }\end{array}$ & C. jejuni & 22 & 14 & D7331 & Empirical & $\begin{array}{l}\text { http://www.outbreakdatabase.com/ } \\
\text { details/hendricks-farm-and-dairy- } \\
\text { raw-milk-2008/ }\end{array}$ \\
\hline $\begin{array}{l}\text { Sprouts } \\
\text { Outbreak }\end{array}$ & E. coli & 10 & 3 & 2011C-3609 & Empirical & $\begin{array}{l}\text { http://www.cdc.gov/ecoli/2014/o12 } \\
\text { 1-05-14/index.html }\end{array}$ \\
\hline $\begin{array}{l}\text { Simulated } \\
\text { outbreak }\end{array}$ & S. enterica & 23 & 18 & CFSAN000189 & Synthetic & $\begin{array}{l}\text { Simulated dataset based off the } S \text {. } \\
\text { enterica spicy tuna outbreak tree } \\
\text { and reference genome. }\end{array}$ \\
\hline
\end{tabular}

$4 \quad{ }^{\text {A }}$ Number of Isolates: Total number of isolates in the dataset

$5 \quad$ B Epidemiologically linked isolates: Number of isolates implicated in the recall or outbreak

$6 \quad{ }^{C}$ Reference genome: suggested reference genome for SNP analysis 


\section{Figure 1 (on next page)}

The "true" phylogeny included for each dataset

The outbreak or event-related taxa are colored red. a) Listeria monocytogenes, b)

Escherichia coli, c) Salmonella enterica, d) Campylobacter jejuni, e) simulated dataset. 


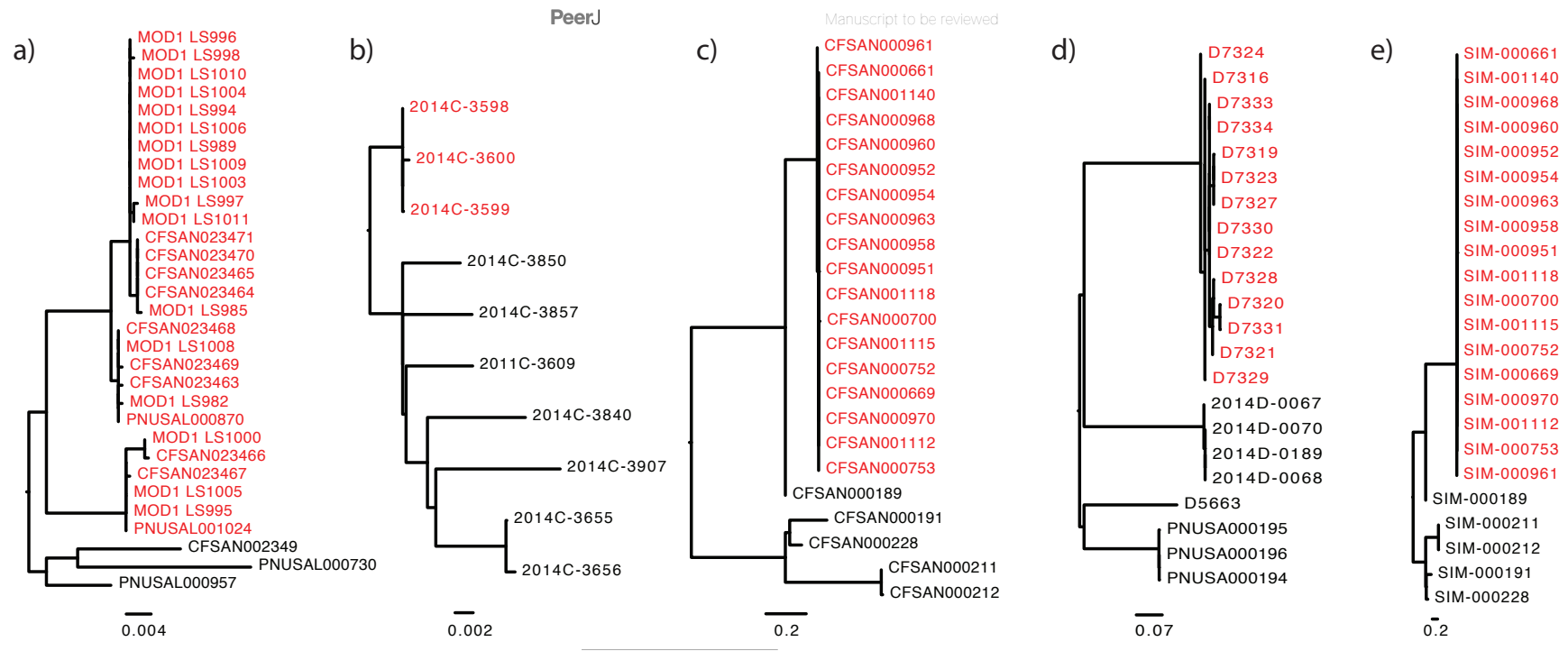

